\title{
SEGREGACIÓN RESIDENCIAL DE LOS EXTRANJEROS EN EL ÁREA METROPOLITANA DE MADRID Un análisis cuantitativo
}

\author{
RESIDENTIAL SEGREGATION OF THE FOREIGN POPULATION IN \\ MADRID'S METROPOLITAN AREA \\ A quantitative analysis
}

\author{
AlFonso ECHAZARRA* \\ CCSR. University of Manchester y CEACS. Instituto Juan March. Madrid. España. \\ aechazarra@ceacs.march.es
}

\begin{abstract}
Resumen
El siguiente artículo se propone tres objetivos. En primer lugar, mostrar la relevancia del lugar de residencia para el desarrollo vital de sus habitantes a través de su relación con los demás espacios de socialización y con los procesos de exclusión social. En segundo lugar, medir la segregación residencial de los extranjeros en el área metropolitana de Madrid en cuatro de las cinco dimensiones propuestas por Denton y Massey (1988), además de utilizar otras medidas menos comunes en la literatura y una nueva medida basada en el indice de disimilitud. Por último, identificar las características de los grupos de extranjeros que se encuentran asociadas a la segregación residencial. En concreto, pretendo averiguar si la segregación urbana está asociada con la situación económica y los rasgos culturales de la población extranjera. El trabajo analiza la distribución residencial de 46 nacionalidades y demuestra que tanto los aspectos culturales como económicos están asociados con la segregación residencial, si bien el trabajo no es capaz de determinar con precisión los mecanismos causales que se esconden detrás de estas correlaciones.
\end{abstract}

\section{Palabras Clave}

Espacios de socialización, Exclusión social, Índices de segregación, Inmigración.

\section{AbstRact}

This article aims at fulfilling three objectives. First of all, stress the importance of neighbourhoods for individual life outcomes, by way of their relationship with other relevant spaces of socialization and with social exclusion processes. Secondly, measure immigrants' residential segregation in Madrid's Metropolitan Area, in four of the five dimensions proposed by Denton and Massey (1988), together with other measures less frequently used

*Agradezco los comentarios y aportaciones de los revisores, así como de los profesores Luis Abad, Joaquín Arango, María Jesús Criado y Asunción Merino. También quisiera reconocer la ayuda del profesor Modesto Escobar con el programa PC-AXIS y la del personal de la biblioteca del Instituto Juan March con los datos de la Encuesta de Población Activa (EPA). 
in the literature and a new measure based on the index of dissimilarity. Finally, find out which variables are associated with the residential segregation. More precisely, identify if the economic and cultural background of foreign nationalities is associated with their residential segregation. The work analyses data for 46 nationalities and demonstrates that both cultural and economic factors are correlated with residential segregation. However, the data does not allow us to discriminate among several causal mechanisms that may lie behind these correlations.

\section{KEYWORDS}

Contexts of Socialization, Immigration, Segregation Indices, Social Exclusion.

\section{INTRODUCCIÓN}

En el otoño de 2005 una ola de violencia sacudió los suburbios franceses. La pobreza, el desempleo juvenil o las implicaciones sociales del origen étnico de los violentos fueron algunas de las explicaciones que se ofrecieron a estos desgraciados incidentes. No obstante, en ninguna de estas tres cuestiones la situación francesa era muy distinta de la de otros países de su entorno. Por el contrario, parecían existir divergencias notables en lo referido a la concentración geográfica de las personas que reúnen conjuntamente las tres características, encontrándose las ciudades francesas en una posición desfavorable frente a la mayoría de ciudades europeas. Aun sin suficientes datos, parece razonable afirmar que la segregación residencial extrema de algunos grupos étnicos, combinada con las excesivas expectativas generadas por una poderosa ideología igualitarista, explica una parte significativa del brote de violencia que se produjo en los suburbios. En sí, la segregación no genera pobreza o desigualdad pero homogeneiza las redes sociales de los individuos, favoreciendo, en el caso de las personas que residen en zonas urbanas degradadas, visiones derrotistas sobre la progresión socioeconómica y, al mismo tiempo, dificultando el disfrute de las conexiones necesarias para un progreso sociolaboral sin esfuerzos sobrehumanos.

Debido principalmente a una planificación urbana mal planteada, aunque posiblemente bien intencionada, los inmigrantes e hijos de inmigrantes de las grandes urbes francesas han acabado fuertemente segregados territorialmente. Este hecho ha reforzado su segregación en la escuela y en el mercado laboral, además de facilitar su identificación como grupo, condición necesaria para la discriminación de tipo geográfico y para la acción colectiva que finalmente se produjo. En definitiva, el problema no parece ser la mera existencia de paro o pobreza sino la concentración territorial de personas que reúnen estas características, creando círculos viciosos que sepultan la esperanza de un progreso socioeconómico. En este sentido, no es casualidad que la segregación residencial sea una de las variables más utilizadas, junto con los matrimonios mixtos y los resultados educativos, a la hora de medir la integración de las personas inmigradas.

En el caso español, el gran número de inmigrantes llegados en los últimos años ha cambiado profundamente la fisonomía de nuestras ciudades, obligándonos a reflexionar sobre la posibilidad de que ocurran revueltas à la française. Sin embargo, el número 
reducido de inmigrantes de segunda generación, cuyas expectativas de progresión socioeconómica rara vez se ven cumplidas, y la moderada aunque creciente concentración de inmigrantes en la escuela, el trabajo y el lugar de residencia parece indicar que, de momento, este tipo de revueltas no van a tener lugar. En cualquier caso, es conveniente observar tendencias de segregación urgentemente ya que el origen de agitaciones como las observadas en ciudades francesas, británicas o americanas se remonta a años o incluso décadas anteriores.

Con el fin de mejorar el conocimiento de la segregación residencial en el contexto español este trabajo se propone tres objetivos: 1) mostrar la relevancia del lugar de residencia para el desarrollo vital de sus habitantes a través de su relación con los demás espacios de socialización y con los procesos de exclusión social, 2) medir la segregación residencial de los extranjeros en el área metropolitana de Madrid y 3) encontrar los factores asociados a esta segregación residencial.

\section{ESPACIOS DE SOCIALIZACIÓN Y EXCLUSIÓN SOCIAL}

La elección del lugar de residencia es una decisión extraordinariamente importante en la vida de las personas. La razón de ello reside en que, en el lugar de residencia, se producen incontables interacciones sociales que influyen, en mayor o menor medida, en la vida de sus habitantes. De hecho, podemos considerar el "barrio", junto a la familia, la escuela y el trabajo, como un espacio de socialización primario donde los individuos conforman buena parte de sus redes sociales. En este sentido, trabajos como los de Musterd y Ostendorf (2007), para el caso holandés, o Goldhaber y Schnell (2007), para el caso de Israel, han demostrado que la proporción que representa un grupo étnico en un barrio limita el contacto con miembros de otros grupos étnicos, aunque nunca de forma determinística.

La importancia del lugar de residencia, no obstante, no sólo consiste en ser un entorno donde los individuos, especialmente los de menor edad, forjan y mantienen relaciones más o menos estrechas sino también en la fuerte interrelación que guarda con los demás espacios de socialización, principalmente la escuela y el trabajo. ${ }^{1}$ Los tres espacios se entrelazan de tal forma que lo que acontece en uno de ellos afecta considerablemente a los demás, hasta tal punto que, en determinadas ocasiones, la red se transforma en un círculo de relaciones que los individuos raramente abandonan. Este círculo puede ser virtuoso, aunque frecuentemente es un círculo vicioso, donde coinciden zonas urbanas deprimidas, altas tasas de paro y escuelas con resultados educativos inferiores a la media. ${ }^{2}$ El gueto hipersegregado, descrito por Massey y Denton

\footnotetext{
${ }^{1}$ Por ejemplo, el lugar de residencia determina, en buena medida, la escuela a la que acuden los alumnos.

${ }^{2}$ La relación entre nivel económico y resultados educativos es evidente en el caso madrileño, donde los centros educativos con mejores notas en selectividad, están sitos en municipios con rentas per capita elevadas como Tres Cantos, Torrelodones, Majadahonda o Pozuelo (Magisnet, 2003).
} 
(1993), la infraclase de la inner city, a la que se refiere Wilson $(1987,1996)$ o el Parque Ansaldo, ilustrado por Martínez Veiga (1999), serían los casos extremos de esta realidad.

La interdependencia entre los tres ámbitos de socialización ayuda a explicar fenómenos como la economía étnica - donde pueden converger empresarios, proveedores, trabajadores, productos y clientes de una misma comunidad étnica- el éxito de las escuelas privadas y concertadas entre la sociedad mayoritaria, el creciente "escapismo" residencial con el fin de limitar la presencia de inmigrantes en las redes sociales propias y de los descendientes y, sobre todo, la exclusión social, entendida como la incapacidad de determinados individuos de materializar su potencial como miembros de una sociedad o como la ausencia de oportunidades de acceso a mercados vitales para el desarrollo humano (residencial, laboral, educativo-formativo).

Este tipo de cuestiones son las que analiza la "nueva segregación" que se centra, principalmente, en los procesos de segregación de personas con bajo estatus socioeconómico (segregación "inferior") (Goldberg, 1998) y su concentración en guetos. En concreto, el círculo vicioso en el que estos grupos se desenvuelven y que limitan sus oportunidades educativas, laborales y de acceso a la vivienda. Sin embargo, y como ha señalado Massey (1996), también es necesario investigar la segregación en ciudadelas de personas con un estatus socioeconómico elevado (segregación "superior") y los círculos virtuosos en los que se desenvuelven, especialmente si tenemos en cuenta que su desahogo económico les permite mayor libertad de elección.

La conexión entre segregación residencial y exclusión social se puede apreciar en la Comunicación de la Comisión Europea sobre Inmigración, integración y empleo donde se señala que:

\begin{abstract}
Varios factores de carácter "involuntario o voluntario"- como la oportunidad de obtener vivienda y trabajo, el deseo de vivir cerca de familiares y de mantener lazos familiares o la discriminación - son responsables de esta situación, que conduce a numerosos inmigrantes a vivir en zonas urbanas deprimidas. La concentración étnica, o los denominados guetos, tiende a aislar a las comunidades y a impedir su participación en la sociedad general. Aunque estrechas redes comunitarias podrían aportar nueva vida y regeneración a barrios descuidados - por ejemplo, con la creación de pequeñas empresas- la segregación étnica y social en las ciudades puede ser también un gran obstáculo a la integración. (COM, 2003-336).
\end{abstract}

No obstante, profundizar en la forma concreta en la que estos tres espacios de socialización se relacionan es una tarea muy compleja que no llevaremos a cabo en este trabajo. Compleja porque en estos ámbitos existen un gran número de actores y de un carácter muy variado, que incluye a padres, profesores, urbanistas, empresarios, propietarios, colectivos de inmigrantes, políticos, etc. Compleja, también, porque los mecanismos de acceso a la vivienda, a la escuela y al trabajo varían considerablemente, alterando la importancia de los distintos actores implicados y la influencia que unos ámbitos ejercen en los demás. 
En cualquier caso, es importante señalar que la relevancia de la segregación residencial no se reduce exclusivamente a su relación con el resto de espacios de socialización sino que es relevante en sí misma, en cuanto que puede afectar negativamente a la cohesión social.

Mi propósito en el resto del artículo será tratar dos cuestiones concretas referidas a uno de los espacios públicos de socialización más relevantes: el lugar de residencia. En primer lugar, trataré de medir la segregación urbana de 46 nacionalidades extranjeras por medio de índices que cubren cuatro de las cinco dimensiones comúnmente utilizadas en la literatura (Massey y Denton 1988), así como la calidad geográfica de los barrios en los que residen y la segregación absoluta en el Área Metropolitana de Madrid (AMM) (Johnston et al. 2003).

En segundo lugar, intentaré encontrar las variables que se asocian a la segregación geográfica de las distintas nacionalidades.

\section{Medición de LA SEGREGACIÓn RESIDENCIAL}

\section{Decisiones metodológicas}

En el cálculo de los índices de segregación espacial es necesario tomar algunas decisiones metodológicas que afectan, en mayor o menor medida, a los resultados. Entre ellas, la elección del conjunto geográfico, la unidad de superficie, el período de estudio, la fuente de datos y los grupos de extranjeros a observar.

El conjunto geográfico elegido es el área metropolitana de Madrid (AMM), entendiendo por área metropolitana el espacio geográfico en el que existe un mercado de trabajo unificado. El criterio utilizado es arbitrario y, en este trabajo, se ha establecido en menos de 50 kilómetros o menos de 60 minutos hasta el municipio de Madrid. De los 178 municipios de la Comunidad de Madrid (CAM), han sido escogidos 109 que representan más del $98 \%$ de la población total.

La unidad de superficie es la unidad geográfica primaria. Con ciertos límites, cuanto menor sea la unidad de superficie, mayor será la relevancia social de los índices. En el caso español, la unidad de superficie más reducida de la que disponemos datos es la sección censal (similar a los tracts estadounidenses), que raramente sobrepasa los 4.000 habitantes y cuyo tamaño medio en el AMM se sitúa en 1.500 habitantes.

El período estudiado es el 2001-2006, con la excepción del índice de uniformidad que se centrará en el año 2005. A lo largo de este período, la población inmigrada en el AMM ha pasado de representar el $6,8 \%$ de la población total a un $13,3 \%$, por lo que es un período excelente para medir la segregación espacial de los extranjeros y su variación en el tiempo.

Los datos proceden del padrón continúo de habitantes, que no es sólo la única fuente que nos ofrece datos por secciones censales con una periodicidad anual, sino que además la calidad de los datos es notable. Aún así, es necesario tomar los datos con precaución porque, como comúnmente se advierte, "ni son todos los que están, ni están todos los que son". 
En cuanto a los grupos de extranjeros, la selección varía en función del índice utilizado. En cualquier caso, la selección se ha hecho independientemente de la renta y con el criterio de nacionalidad. En futuras investigaciones, y a medida que el número de extranjeros que adquieran la nacionalidad española aumente, será recomendable incorporar el criterio del lugar de nacimiento.

\section{1) Disimilitud (Uniformidad): Índice $D$}

Este ha sido el índice más habitual para medir la segregación residencial y, sin lugar a dudas, el de mayor aceptación para medir la uniformidad, entendida como el grado en que la proporción de miembros de un grupo social en una unidad de superficie (secciones censales, distritos, municipios) se asemeja a la proporción del mismo grupo en el conjunto geográfico analizado (ciudades, áreas metropolitanas, regiones). El índice de disimilitud varía desde 0 hasta 1, donde 0 representa la máxima uniformidad y 1 la máxima disimilitud. La fórmula del índice de uniformidad, que a partir de ahora representaremos por una $D$, es la siguiente:

$$
\text { (1) } D=\sum_{i=1}^{n}\left[t_{i}\left|p_{i}-P\right| / 2 T P(1-P)\right]
$$

donde $t_{i}$ y $p_{i}$ son la población total y la proporción del grupo de interés en la unidad de superficie, y $T$ y $P$ son la población total y la proporción del grupo de interés en el conjunto geográfico analizado, que está dividido en $n$ unidades de superficie.

La fórmula compara la proporción que representa el grupo de estudio en cada una de las unidades de superficie (secciones censales) con la proporción que representa en el conjunto geográfico analizado (área metropolitana de Madrid), corregido de tal manera que el valor adopte valores entre 0 y 1 . Las principales ventajas de este índice son su sencilla obtención y su acotamiento entre los valores 0 y 1 lo que, a priori, facilita su comparación en el tiempo y en el espacio. Sin embargo, este índice es excesivamente sensible al tamaño de la población lo que falsea cualquier tipo de comparación. A pesar de la dificultad para establecer comparaciones apropiadas, este índice se encuentra presente en la mayoría de los trabajos cuantitativos sobre segregación residencial.

Este falseamiento se puede apreciar en las dos tablas siguientes donde se muestran los índices de población de cuatro grupos con tamaños de población muy distintos y los índices de un mismo grupo a lo largo del tiempo.

Los resultados de las tablas 1 y 2 son reveladores. No parece razonable que la segregación de los indios sea el doble que la de los ecuatorianos o que la segregación de los rumanos haya disminuido más de un $30 \%$ en un período de 5 años. La razón de que estos datos no nos sirvan para establecer comparaciones es que $D$, en realidad, mide la proporción de miembros del grupo que deberían cambiar de sección censal 
Tabla 1.

Índice de disimilitud y tamaño de población (2005).

\begin{tabular}{lcr}
\hline Nacionalidad & D & Población \\
\hline Ecuador & 0,411 & 171.102 \\
Marruecos & 0,485 & 64.552 \\
Francia & 0,539 & 9.926 \\
India & 0,861 & 1.203 \\
\hline
\end{tabular}

Unidad de superficie: sección censal.

Datos: Instituto Nacional de Estadística (INE).

Tabla 2.

Índice de disimilitud y tamaño de población (Rumanía).

\begin{tabular}{lcc}
\hline Año & D & Población \\
\hline 2001 & 0,634 & 18.210 \\
2003 & 0,569 & 46.420 \\
2005 & 0,466 & 93.403 \\
2006 & 0,436 & 111.737
\end{tabular}

Unidad de superficie: sección censal.

Datos: Instituto Nacional de Estadística (INE).

para que su población estuviera uniformemente distribuida en toda el área metropolitana (Massey y Denton, 1988) y las proporciones son dependientes del tamaño de la población. Por ejemplo, en el caso de los indios, y a pesar de tener un valor de $D$ muy elevado, sería suficiente para alcanzar la uniformidad si 1.035 personas cambiaran de residencia mientras que en el caso de los ecuatorianos, con un valor de $D$ moderado, serían necesarias 70.322 personas. Por otra parte, el sesgo introducido por el tamaño de población es especialmente grave cuando el número de nacionales de un país es inferior al número total de secciones censales, como ocurre con la población india, moldava 0 bangladeshí durante el período de estudio.

Para subsanar esta deficiencia y poder comparar adecuadamente los distintos grupos he optado por un método consistente en corregir el efecto que el tamaño de la población tiene sobre $D .{ }^{3}$ Es decir, calcular $D$ para los distintos grupos si todos ellos

\footnotetext{
${ }^{3}$ El método se detalla en el apéndice.
} 
tuvieran igual población. ${ }^{4}$ Los valores de este índice, que llamaremos $D$-real, indican mayor segregación a medida que aumentan, siendo valores extremos aquellos inferiores a - 0,10 y mayores a 0,10 . Los resultados de las zonas geográficas y nacionalidades más relevantes, sólo para el año 2005, se muestran en las tablas 3, 4, 5 y 6 .

Tabla 3.

D-real en el AMM (2005): Zonas geográficas.

\begin{tabular}{lrr}
\hline Nacionalidad & D-real & Población \\
\hline UE-25 (1) & $-0,09$ & 69.364 \\
Europa no-UE & 0,03 & 138.210 \\
America N. & 0,00 & 14.000 \\
América C. & $-0,03$ & 35.519 \\
América Sur & $-0,01$ & 364.176 \\
Asia & 0,05 & 42.959 \\
África & 0,05 & 94.733 \\
\hline
\end{tabular}

Unidad de superficie: sección censal.

Datos: INE.

(1) Sin España

Tabla 4.

D-real en el AMM (2005): Nacionalidades con mayor población.

\begin{tabular}{lrr}
\hline Nacionalidad & D-real & Población \\
\hline Ecuador & 0,16 & 171.102 \\
Rumanía & 0,14 & 93.403 \\
Colombia & $-0,04$ & 93.403 \\
Marruecos & 0,12 & 64.552 \\
Perú & $-0,05$ & 38.910 \\
Bolivia & 0,02 & 26.031 \\
China & 0,10 & 23.922 \\
\hline
\end{tabular}

Unidad de superficie: sección censal.

Datos: INE.

${ }^{4}$ La comparación de los valores de este índice a través del tiempo y espacio será posible en el caso de que el efecto del tamaño de la población sobre el índice sea muy parecido en otros contextos. La probabilidad de que esta condición ocurra es alta debido a que el efecto de la población se explica, fundamentalmente, por las propiedades del índice, que son independientes del contexto. 
Tabla 5.

D-real en el AMM (2005): Nacionalidades con valores más bajos.

\begin{tabular}{lcc}
\hline Nacionalidad & D-real & Población \\
\hline Cuba & $-0,14$ & 8.441 \\
Portugal & $-0,14$ & 8.803 \\
Argentina & $-0,13$ & 20.661 \\
\hline
\end{tabular}

Unidad de superficie: sección censal.

Datos: INE.

Tabla 6.

D-real en el AMM (2005): Nacionalidades con valores más altos.

\begin{tabular}{lcr}
\hline Nacionalidad & D-real & Población \\
\hline Bangladesh & 0,17 & 1.931 \\
Ecuador & 0,16 & 171.102 \\
Rumanía & 0,14 & 93.403 \\
\hline
\end{tabular}

Unidad de superficie: sección censal.

Datos: INE.

Con el índice $D$-real es posible comparar los datos por nacionalidades ya que podemos considerar que este nuevo índice mide adecuadamente la segregación en la dimensión de la uniformidad. Por zonas geográficas, la UE-25 presenta la menor segregación, mientras que Asia y África la mayor. En cuanto a las nacionalidades con mayor población -entre todas ellas representan más del $60 \%$ de la población inmigrada en la CAM-hay que destacar la fuerte segregación de ecuatorianos, rumanos, marroquíes y chinos y la reducida segregación de colombianos y peruanos. Por último, respecto a los valores extremos, los cubanos, portugueses y argentinos presentan la segregación más baja, mientras que los nacionales de Bangladesh, Ecuador y Rumanía la más alta.

Los valores de $D$, hasta ahora presentados, son la suma de la segregación debida a las secciones censales, los distritos y los municipios. Sin embargo, la relevancia de la segregación es distinta en función del nivel geográfico al que atribuyamos la segregación. Desde mi punto de vista, la relevancia de la segregación es mayor cuando ésta se debe a unidades geográficas muy reducidas, como la sección censal, y menor cuando se debe a unidades geográficas más extensas, como el municipio. Esto es así por dos razones. En primer lugar, porque en Madrid no existen municipios que podamos considerar deprimidos en su totalidad pero sí existen secciones censales reconocidamente degradadas. También, porque las relaciones "de barrio" se establecen generalmente en unidades geográficas reducidas (por ejemplo, una calle, una plaza...), que no coinciden exactamente pero se aproximan más a las secciones censales que a municipios o distritos enteros. 
Imaginemos dos grupos de extranjeros. El primero se encuentra uniformemente distribuido entre municipios y distritos. No obstante, dentro de los distritos todos los individuos del grupo residen en la misma sección censal (Cuadro 1e). El segundo grupo se encuentra presente en muy pocos municipios o distritos y, sin embargo, dentro de los pocos distritos o municipios en los que habita los individuos del grupo están perfectamente mezclados con la población local (Cuadro 1c o 1d). ¿Qué tipo de segregación estará asociada, en mayor medida, con prácticas de exclusión social? Probablemente, el primer grupo puesto que la probabilidad de que establezca redes sociales extensas y heterogéneas serán menores.

Los distintos tipos de segregación, en relación a las unidades geográficas - municipios, distritos y secciones- explicativas de esta segregación, se muestran en los Cuadros 1a a $1 f$.

Aunque los tipos de distribución territorial mostrados en el Cuadro 1 sean hipotéticos, pueden servirnos de ayuda para reflejar el tipo de segregación de las distintas nacionalidades. Por ejemplo, la segregación residencial de los rumanos y ecuatorianos se explica principalmente por su desigual distribución en municipios (Cuadro 1c; concentración en el este de la CAM los primeros y en el sur del municipio de Madrid los segundos), mientras que en el caso de los hondureños, rusos o argelinos es su desigual distribución en secciones (Cuadro 1e).

Con el fin de asociar de forma más sólida la segregación residencial con la exclusión social, he creado un nuevo índice, a partir de $D$-real, que mide la uniformidad real que se debe exclusivamente a las secciones censales. Los valores, junto con los de $D$-real, se pueden observar en las tablas 7,8 y 9 .

Los cambios en los valores de los dos índices son significativos para la mayoría de las nacionalidades. Los nuevos valores presentan una mayor asociación con la renta económica, lo que apunta a una relación mas intensa entre exclusión social y segrega-

Tabla 7.

D-real en el AMM (2005): Nacionalidades con valores más altos.

\begin{tabular}{lcc}
\hline Nacionalidad & D-real & D-real secciones \\
\hline Ecuador & 0,16 & 0,09 \\
Rumanía & 0,14 & 0,01 \\
Colombia & $-0,04$ & 0,05 \\
Marruecos & 0,12 & 0,06 \\
Perú & $-0,05$ & 0,01 \\
Bolivia & 0,02 & 0,02 \\
China & 0,10 & 0,05
\end{tabular}

Unidad de superficie: sección censal.

Datos: INE. 


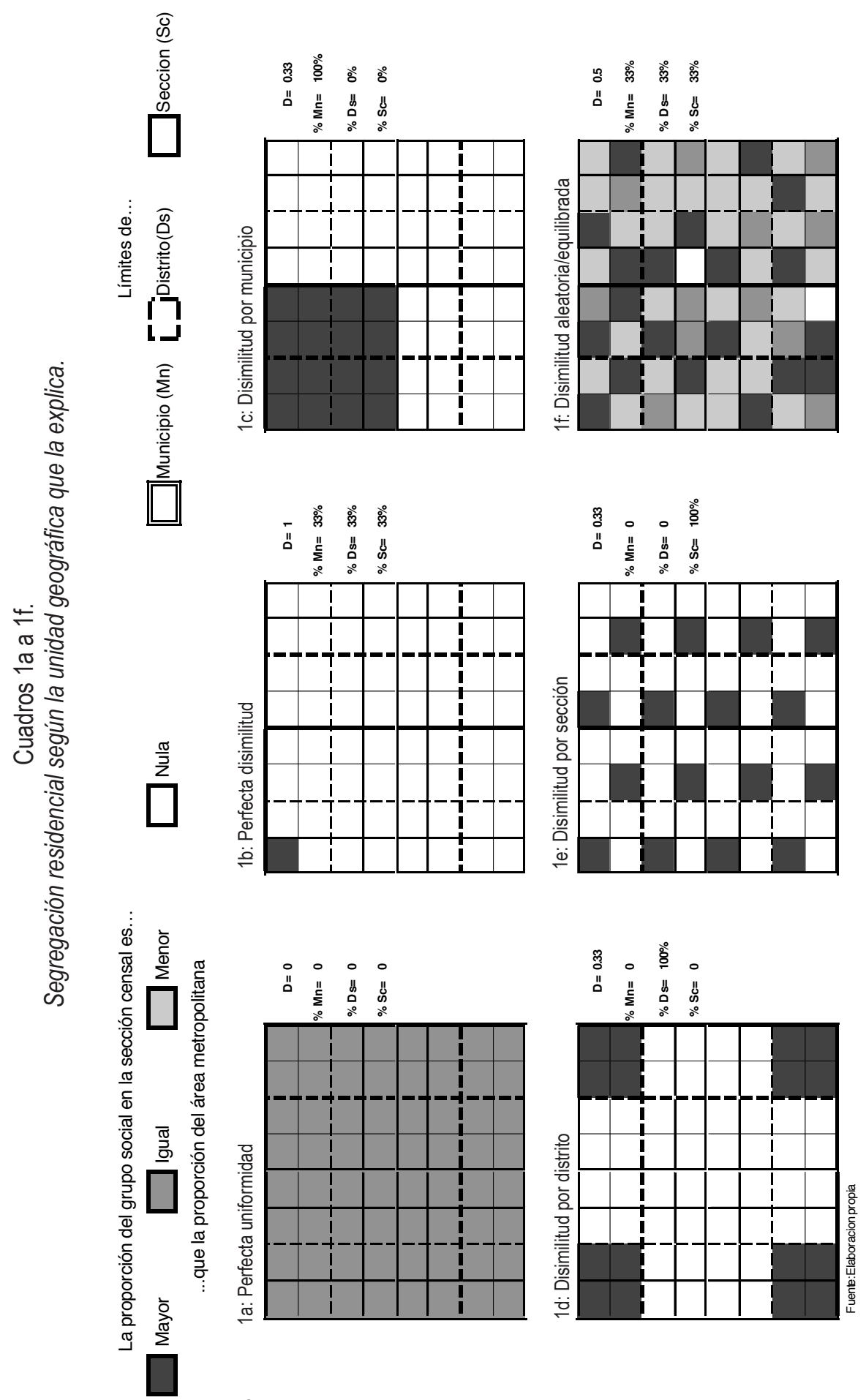

RIS, VOL. 68, NN 1, ENERO-ABRIL, 165-197, 2010. ISSN: 0034-9712. DOI: 10.3989/ris.2007.11.12 
Tabla 8.

D-real secciones: Nacionalidades con valores más bajos.

\begin{tabular}{lcc}
\hline Nacionalidad & D-real & D-real secciones \\
\hline Bangladesh & 0,17 & $-0,19$ \\
Reino Unido & $-0,09$ & $-0,14$ \\
Alemania & $-0,09$ & $-0,13$ \\
Francia & $-0,05$ & $-0,11$ \\
EEUU & $-0,09$ & $-0,11$ \\
\hline
\end{tabular}

Unidad de superficie: sección censal.

Datos: INE.

ción residencial cuanto más reducido es el nivel geográfico. Esto es lo que ilustran las nacionalidades con mayores rentas que España, cuya posición media, en base a un ranking que va de menor a mayor segregación residencial, pasa de la $14^{a}$, con $D$-real, a la $9^{a}$ con el nuevo índice basado en las secciones censales.

La elaboración del nuevo índice nos permite, además, determinar la contribución de cada nivel geográfico - municipios, distritos y secciones censales - a la segregación residencial. En términos generales, esta contribución es equilibrada en el caso de los países con mayores rentas y muy desequilibrada en el caso de los países con menores rentas. Por ejemplo, en los casos de Rumania o Nigeria, más de la mitad de la segregación residencial la explica su desigual distribución por municipios (Cuadro 1c), en los casos de Honduras, Rusia, Irán o Argelia, más de la mitad su desigual distribución por secciones censales (Cuadro 1e), mientras que en los casos de EEUU, Alemania, Italia o Reino Unido, los tres niveles explican entre un $30 \%$ y un $40 \%$ cada uno (Cuadro 1 ).

Tabla 9.

D-real secciones: Nacionalidades con valores más altos.

\begin{tabular}{lcc}
\hline Nacionalidad & D-real & D-real secciones \\
\hline Honduras & 0,01 & \\
Irán & 0,01 & 0,15 \\
Cabo Verde & 0,02 & 0,12 \\
Argelia & 0,01 & 0,11 \\
Ecuador & 0,16 & 0,11 \\
\end{tabular}

Unidad de superficie: sección censal.

Datos: INE. 
En definitiva, las nacionalidades con menores rentas no sólo presentan mayor segregación, sino también una segregación muy desequilibrada, que se debe fundamentalmente a su desigual distribución por municipios o secciones.

\section{2) Exposición: índice $x P^{*} y$}

Menos común en los estudios de segregación, el índice de exposición mide la probabilidad de que los miembros de dos grupos habiten en una misma zona residencial (en nuestro caso, secciones censales). 0 , desde otro punto de vista, si los contactos entre personas se establecieran exclusivamente en el lugar de residencia, el índice mediría la probabilidad de que los miembros de dos grupos estableciesen contactos.

El índice está acotado entre 0 y 1 , aumentando el potencial de contacto entre dos grupos a medida que el índice se acerca a 1 y disminuyendo al acercarse a $0 .{ }^{5} \mathrm{El}$ índice de exposición es muy sensible al tamaño del grupo de referencia por lo que, a la hora de efectuar comparaciones, conviene medirlo no sólo términos absolutos sino también en términos relativos, controlando por la proporción que la población de referencia representa en el conjunto geográfico. Los valores relativos positivos representan mayor exposición que la esperada de una distribución aleatoria de la población de interés, siendo el valor 0 el valor esperado de una distribución aleatoria.

El índice ha sido generalmente utilizado para medir la exposición de un grupo minoritario al grupo mayoritario (por ejemplo, exposición de ecuatorianos y marroquíes a españoles), si bien es posible medir la exposición de cualesquiera dos grupos, incluso la exposición de un grupo a los miembros de ese mismo grupo. ${ }^{6}$ En este sentido, y como se hará posteriormente, puede ser interesante comparar la exposición de unas nacionalidades a otras con el fin de conocer las pautas de agrupamiento entre distintas nacionalidades.

La fórmula del índice de exposición, que a partir de ahora representaremos por una $x P^{*} y$, es la siguiente:

(2) $x P * y=\sum_{i=1}^{n}\left[x_{i} / X\right]\left[y_{i} / t_{i}\right]$

donde $x_{i}$ e $y_{i}$ son el número de miembros de los grupos $X$ e $Y$ en la unidad de superficie $i$ (secciones censales), $t_{i}$ es la población total de la unidad de superficie $i$, y $X$ es la población total del grupo $X$ en todo el conjunto geográfico (área metropolitana).

\footnotetext{
${ }^{5}$ En este trabajo se ha acotado entre 0 y 100 para facilitar su interpretación.

${ }^{6}$ En la literatura se llama "índice de aislamiento" y mide la probabilidad de que los miembros de un grupo residan en la misma zona residencial.
} 
Los valores absolutos y relativos de $x P^{*} y$, tomando como grupo de referencia la población con nacionalidad española, y sólo para las nacionalidades más importantes dentro de cada zona geográfica, aparecen en la tablas 10 y 11.

Como se puede observar en la tabla 10, los valores absolutos del índice van disminuyendo a lo largo del tiempo para todos los grupos. Es decir, a medida que pasa el tiempo, la probabilidad de convivir en la misma sección censal con una persona con nacionalidad española disminuye para todas las nacionalidades, también para la española. La razón principal de ello es que la población española representa, cada año, una proporción menor de la población total (de 93,2\% en el año 2001 a 86,7\% en el 2006). Sin embargo, los valores relativos de la tabla 11 apuntan a que existen, además, otros factores que han contribuido a la disminución de la exposición de los extranjeros a los españoles. ${ }^{7} \mathrm{De}$ hecho, si la proporción de españoles (o extranjeros) en el AMM se hubiera mantenido estable a lo largo de todo el período, podríamos afirmar que los españoles "se juntan cada vez más entre ellos" y los extranjeros "se juntan cada vez menos con españoles". Por desgracia, los datos no nos permiten conocer cuáles son los factores que están afectando a la menor convivencia, en términos relativos, entre españoles y extranjeros.

Tabla 10.

Índice de Exposición absoluto a los españoles en el AMM.

\begin{tabular}{lcccc}
\hline Nacionalidad & 2001 & 2003 & 2005 & 2006 \\
\hline España (Aislamiento: xP*x) & 93,5 & 90,1 & 87,8 & 87,4 \\
Extranjera & 89,3 & 85,0 & 82,1 & 81,7 \\
UE-25 & 90,9 & 87,2 & 84,9 & 84,8 \\
UE-15 & 90,5 & 87,2 & 85,2 & - \\
Europa no-UE & 90,6 & 86,0 & 82,6 & 81,8 \\
Rumanía & 91,0 & 86,0 & 82,4 & 81,4 \\
África & 90,3 & 85,5 & 81,7 & 81,0 \\
Marruecos & 89,9 & 85,0 & 81,6 & 81,0 \\
América Central & 88,8 & 84,1 & 81,3 & - \\
República Dominicana & 88,1 & 83,2 & 80,1 & - \\
América del Sur & 88,5 & 84,3 & 81,6 & - \\
Ecuador & 87,0 & 82,7 & 80,2 & 80,1 \\
Asia & 88,3 & 83,5 & 80,2 & 80,1 \\
China & 88,6 & 83,5 & 80,1 & 80,2 \\
\% España en AMM & 93,2 & 89,6 & 87,0 & 86,7 \\
\hline
\end{tabular}

Unidad de superficie: sección censal

Datos: INE. En negrita valores máximos y mínimos para cada año

${ }^{7}$ Con la excepción de la UE-15 y la UE-25. 
Tabla 11.

Índice de Exposición relativo a los españoles en el AMM.

\begin{tabular}{lcccc}
\hline Nacionalidad & 2001 & 2003 & 2005 & 2006 \\
\hline España (Aislamiento: P $^{*} \mathrm{x}$ ) & 0,3 & 0,5 & 0,8 & 0,7 \\
Extranjera & $-3,9$ & $-4,6$ & $-4,9$ & $-5,0$ \\
UE-25 & $-2,3$ & $-2,4$ & $-2,1$ & $-1,9$ \\
UE-15 & $-2,7$ & $-2,4$ & $-1,8$ & - \\
Europa no-UE & $-2,6$ & $-3,6$ & $-4,4$ & $-4,9$ \\
Rumanía & $-2,2$ & $-3,6$ & $-4,6$ & $-5,3$ \\
África & $-2,9$ & $-4,1$ & $-5,3$ & $-5,7$ \\
Marruecos & $-3,3$ & $-4,6$ & $-5,4$ & $-5,7$ \\
América Central & $-4,4$ & $-5,5$ & $-5,7$ & - \\
República Dominicana & $-5,1$ & $-6,4$ & $-6,9$ & - \\
América del Sur & $-4,7$ & $-5,3$ & $-5,4$ & - \\
Ecuador & $-6,2$ & $-6,9$ & $-6,8$ & $-6,6$ \\
Asia & $-4,9$ & $-6,1$ & $-6,8$ & $-6,6$ \\
China & $-4,6$ & $-6,1$ & $-6,9$ & $-6,5$ \\
\hline
\end{tabular}

Unidad de superficie: sección censal.

Datos: INE. En negrita valores máximos y mínimos para cada año.

Por nacionalidades, lo más relevante es la baja exposición de ecuatorianos, chinos y dominicanos, la alta y creciente exposición relativa de personas con nacionalidad de un país de la UE y la fuertemente decreciente exposición relativa de rumanos. En resumen, los valores del índice de exposición no sólo muestran una fuerte segregación "inferior", encarnada por inmigrantes con un bajo nivel socioeconómico, sino que esta segregación parece acentuarse a lo largo del período (con la excepción de Ecuador).

Hasta ahora, hemos utilizado el índice de exposición de algunas nacionalidades a los ciudadanos españoles. Sin embargo, y como apunté anteriormente, es posible indagar en las pautas de agrupamiento entre grupos de extranjeros por medio de $x P^{*} y$. La cantidad de valores que podemos obtener es una matriz de $n^{*} n$ donde $n$ es el número de nacionalidades. Al ser una matriz de 46 nacionalidades excesivamente grande, he optado por mostrar una matriz de valores de $x P^{*} y$ que nos informe de la influencia que puede ejercer la cercanía cultural a la hora de agruparse geográficamente. En la tabla 12 , se presenta una matriz de datos donde las nacionalidades cercanas culturalmente están agrupadas por parejas. ${ }^{8} \mathrm{Si}$ la exposición de una nacionalidad a su pareja cultural es superior a la del resto de nacionalidades, la cercanía cultural puede ser un factor explicativo de la segregación residencial.

\footnotetext{
${ }^{8}$ Una pareja por zona geográfica.
} 
En la tabla 12, que se lee por columnas, podemos observar como para todas las nacionalidades, la pareja cultural es la primera nacionalidad más expuesta (en 7 de 10 casos) o la segunda (en 2 de 10 casos), excepto en el caso de la nacionalidad ecuatoriana, cuya pareja cultural (Perú) es la tercera. Como era de esperar, el índice de aislamiento -la exposición de un grupo al mismo grupo- es el valor más alto en todos los casos, con la excepción de Ecuador. Sorprendentemente, la probabilidad de convivir con un ecuatoriano, en la misma sección censal, es más alta para los nacionales de Bangladesh que para los propios ecuatorianos.

Estos datos confirman parcialmente la hipótesis del efecto cultural en la segregación. Parcialmente porque, aunque los datos sean capaces de descartar explicaciones relacionadas con redes previas a la emigración, no descarta la explicación económica, sobre todo si tenemos en cuenta que la afinidad cultural y el nivel adquisitivo de los grupos de extranjeros están fuertemente correlacionados. En la última parte del trabajo intentaré separar ambos efectos.

\section{3) Centralización: índices CTM y CEM}

En este trabajo se emplean dos índices para medir la centralización, entendida como el grado en el que un grupo se localiza cerca del núcleo urbano (Massey y Denton 1988). Estos índices, que denomino índice de centralización temporal media (CTM) e índice de centralización espacial media (CEM), miden el tiempo (en vehículo de motor) y la distancia media recorrida hasta el centro urbano. El principal inconveniente de estos índices es que no están acotados por lo que las comparaciones con otros contextos no son sencillas. Una posibilidad, que se lleva a cabo en este trabajo, es comparar la distancia y el tiempo medio de un grupo con el del grupo mayoritario, convirtiéndolo así en un valor relativo fácilmente comparable entre contextos.

Las fórmulas de los índices son las siguientes:

(3) $C T M=\sum_{i=1}^{n}\left[x_{i} / X\right] m_{i}$

(4) $C E M=\sum_{i=1}^{n}\left[x_{i} / X\right] d_{i}$

donde $x_{i}$ y $X$ son la población del grupo $X$ en la unidad de superficie $i$ y en el conjunto geográfico respectivamente y $m_{i}$ y $d_{i}$ son el tiempo y la distancia desde la unidad de superficie $i$. Los valores de CEM y CTM, tomando el municipio (y no la sección censal) como unidad de superficie, se muestran en las tablas 13 y 14.

Las tablas muestran tres tendencias muy interesantes. En primer lugar, los extranjeros se sitúan más cercanos al centro urbano que los españoles, con la excepción de los 


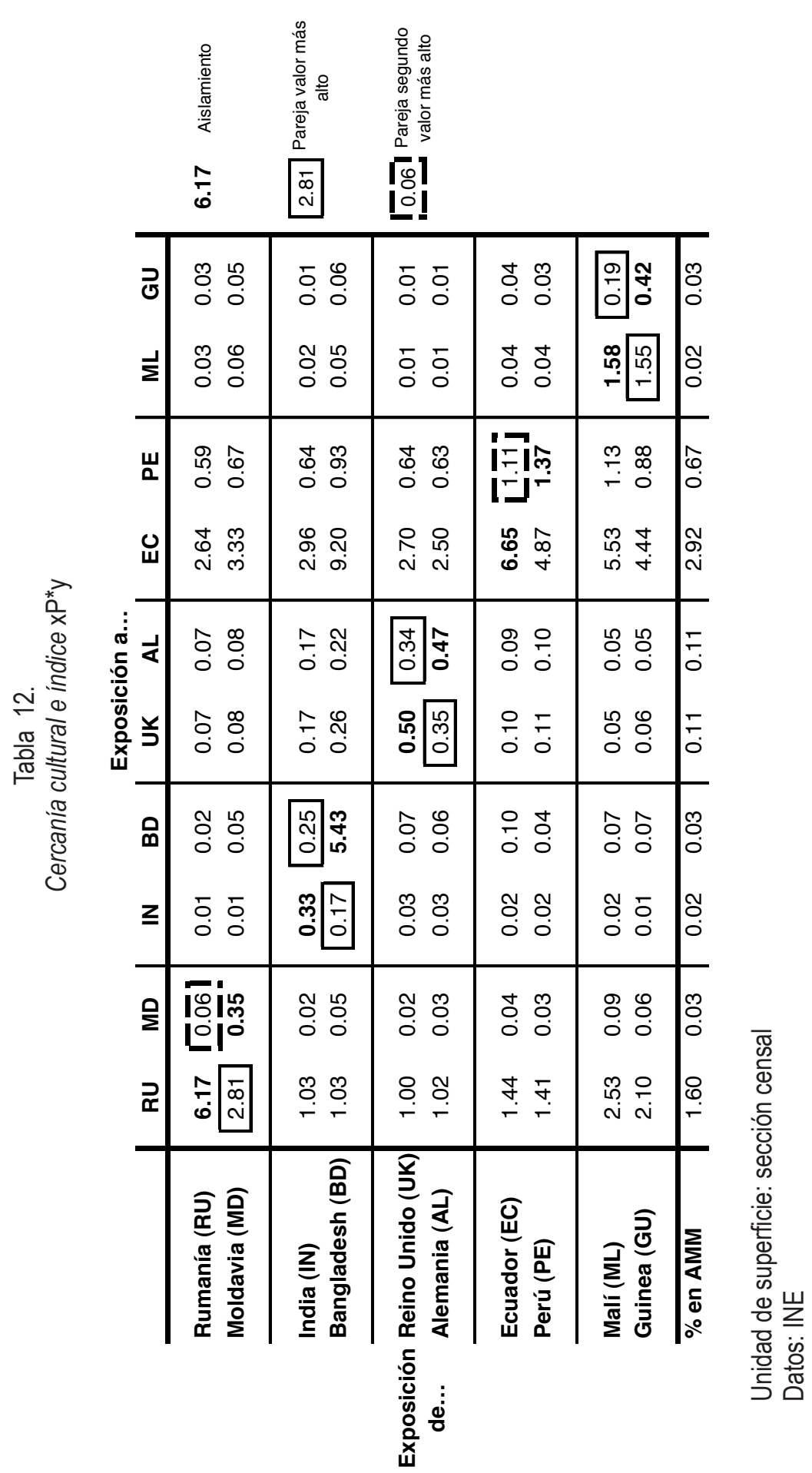

RIS, VOL. 68, No 1, ENERO-ABRIL, 165-197, 2010. ISSN: 0034-9712. DOI: 10.3989/ris.2007.11.12 
rumanos y marroquíes. ${ }^{9}$ En segundo lugar, durante el período 2001-2006, la distancia al centro urbano ha aumentado de forma generalizada. Por último, se aprecian mayores cambios en los valores a lo largo del tiempo en las comunidades con períodos de residencia más cortos. Por ejemplo, los marroquíes y dominicanos, con períodos de residencia relativamente largos, prácticamente no varían sus valores en todo el período, mientras que los rumanos y ecuatorianos, con períodos de residencia más cortos, los aumentan considerablemente. Algunas de las razones que podrían explicar estas tendencias están asociadas con la mayor presencia de redes de inmigrantes en el centro urbano (primera tendencia), el aumento del diferencial de precios centro-periferia y la saturación residencial del centro urbano (segunda tendencia) y la mayor movilidad laboral y residencial de las personas recién llegadas (tercera tendencia).

Tabla 13.

CEM en el AMM: kilómetros al centro* y valores relativos.

\begin{tabular}{llllc}
\hline Nacionalidad & \multicolumn{1}{c}{2001} & \multicolumn{2}{c}{2003} & \multicolumn{2}{c}{2005} & 2006 \\
\hline Española & 14,0 & 14,3 & 14,6 & 14,8 \\
Extranjera & $12,4(0,89)$ & $13,3(0,93)$ & $13,9(0,95)$ & $14,4(0,98)$ \\
UE-25 & $14,9(1,06)$ & $15,2(1,06)$ & $15,0(1,02)$ & $15,2(1,03)$ \\
UE-15 & $13,8(0,99)$ & $14,1(0,98)$ & $13,9(0,94)$ & - \\
Europa no-UE & $16,9(1,21)$ & $18,8(1,31)$ & $19,2(1,31)$ & $19,7(1,33)$ \\
Rumanía & $17,6(1,26)$ & $19,8(1,38)$ & $20,1(1,37)$ & $20,5(1,39)$ \\
África & $17,6(1,25)$ & $18,2(1,27)$ & $18,7(1,27)$ & $19,1(1,29)$ \\
Marruecos & $19,0(1,36)$ & $19,6(1,37)$ & $20,0(1,36)$ & $20,2(1,37)$ \\
América Central & $10,8(0,77)$ & $11,0(0,77)$ & $11,2(0,76)$ & - \\
República Dominicana & $10,5(0,75)$ & $10,5(0,73)$ & $10,6(0,72)$ & - \\
América del Sur & $9,8(0,70)$ & $10,9(0,76)$ & $11,3(0,77)$ & $11,6(0,79)^{* *}$ \\
Ecuador & $7,8(0,56)$ & $8,9(0,62)$ & $9,7(0,66)$ & $10,1(0,68)$ \\
Asia & $9,6(0,69)$ & $9,7(0,68)$ & $9,5(0,64)$ & $9,9(0,67)$ \\
China & $9,7(0,69)$ & $9,8(0,69)$ & $9,4(0,64)$ & $10,0(0,67)$ \\
\hline
\end{tabular}

*Centro: plaza Cibeles

En negrita valores máximos y mínimos para cada año

${ }^{* *}$ Continente americano

Valores relativos en paréntesis; Nacionalidad española $=1,00$

Unidad de superficie: municipio.

Datos: INE.

${ }^{9}$ Además de otras nacionalidades, que no se encuentran en la tabla, como la moldava o la polaca. 
Asimismo, los datos sugieren que los latinoamericanos y europeos no-UE recién llegados se instalan preferentemente en el centro y, a medida que mejoran su conocimiento del mercado inmobiliario local, se estabilizan económicamente y amplían sus redes sociales, se extienden por todo el conjunto geográfico. ${ }^{10}$

\section{4) Concentración: índice DM}

La concentración se refiere a la cantidad de espacio físico ocupado por un grupo en un entorno urbano (Massey y Denton; 1988). Existen diversos índices para medir la concentración. En este trabajo he optado por el más sencillo de ellos: el índice de densidad media $(D M)$, que funciona con la misma lógica y posee las mismas propiedades que los índices de centralización empleados anteriormente. Su fórmula es la siguiente:

(5) $C T M=\sum_{i=1}^{n}\left[x_{i} / X\right] d_{i}$

donde $x_{i}$ y $X$ representan lo mismo que en los índices de centralización y $d_{i}$ es la densidad de la unidad de superficie $i$.

Los valores para el período 2001-2005, tomando otra vez las secciones como unidad de superficie, aparecen en la tabla 15.

Los datos muestran que los nacionales de los países miembros de la Unión Europea son los que viven en áreas residenciales más descongestionadas, mientras que los americanos y asiáticos son los que viven en áreas más densamente pobladas. En cuanto a los cambios a lo largo del período, parece claro que la densidad media ha aumentado considerablemente para todas las nacionalidades, debido a que la población total del AMM ha pasado de menos de cinco millones y medio en el año 2001 a casi seis millones en el año 2005.

\section{5) Calidad geográfica de los barrios}

Utilizando los índices de concentración y centralización es posible crear un nuevo índice que mida la calidad geográfica o de localización de las zonas donde residen los extranjeros. El índice se calcula hallando la media entre los valores relativos de la distancia media y de la densidad media, considerando que la calidad geográfica del lugar de residencia aumenta a medida que la distancia al centro urbano y la densidad de población

\footnotetext{
${ }^{10}$ Para confirmar esta hipótesis y evitar caer en una falacia ecológica -explicar comportamientos individuales en base a datos agregados- serian necesarios datos individuales que mostraran las trayectorias residenciales de los extranjeros.
} 
Tabla 14.

CTM en el AMM: minutos al centro* $y$ valores relativos.

\begin{tabular}{lcccc}
\hline Nacionalidad & 2001 & 2003 & 2005 & 2006 \\
\hline Española & 17,8 & 18,0 & 18,3 & 18,4 \\
Extranjera & $16,4(0,92)$ & $17,1(0,95)$ & $17,6(0,96)$ & $18,0(0,98)$ \\
UE-25 & $18,6(1,05)$ & $18,8(1,04)$ & $18,6(1,01)$ & $18,7(1,02)$ \\
UE-15 & $17,7(1,00)$ & $17,9(1,00)$ & $17,7(0,97)$ & - \\
Europa no-UE & $19,9(1,12)$ & $21,4(1,19)$ & $21,7(1,19)$ & $22,1(1,20)$ \\
Rumanía & $20,3(1,14)$ & $22,0(1,22)$ & $22,3(1,22)$ & $22,7(1,23)$ \\
África & $20,8(1,17)$ & $21,3(1,18)$ & $21,6(1,18)$ & $22(1,20)$ \\
Marruecos & $21,9(1,23)$ & $22,3(1,23)$ & $22,7(1,24)$ & $22,9(1,25)$ \\
América Central & $15,2(0,85)$ & $15,3(0,85)$ & $15,4(0,84)$ & - \\
República Dominicana & $14,9(0,84)$ & $14,9(0,83)$ & $15,0(0,82)$ & - \\
América del Sur & $14,2(0,80)$ & $15,1(0,84)$ & $15,4(0,85)$ & $15,8(0,86)^{\star *}$ \\
Ecuador & $12,5(0,70)$ & $13,5(0,75)$ & $14,1(0,77)$ & $14,5(0,79)$ \\
Asia & $14,1(0,79)$ & $14,2(0,79)$ & $13,9(0,76)$ & $14,3(0,78)$ \\
China & $14,1(0,80)$ & $14,2(0,79)$ & $13,9(0,76)$ & $14,4(0,78)$ \\
\hline
\end{tabular}

*Centro: plaza Cibeles

En negrita valores máximos y mínimos para cada año

${ }^{* *}$ Continente americano

Valores relativos en paréntesis; Nacionalidad española $=1,00$

Unidad de superficie: municipio.

Datos: INE

Tabla 15.

$D M$ en el AMM: miles de habitantes por $\mathrm{km}^{2}$ y valores relativos.

\begin{tabular}{lccc}
\hline Nacionalidad & 2001 & 2003 & 2005 \\
\hline España & 27,3 & 29,8 & 28,7 \\
Extranjera & $28,1(1,07)$ & $34,8(1,17)$ & $34,2(1,19)$ \\
UE-25 & $22,6(0,83)$ & $26,8(0,90)$ & $27,3(0,95)$ \\
UE-15 & $20,0(0,73)$ & $23,7(0,80)$ & $24,8(0,86)$ \\
Europa no-UE & $28,2(1,04)$ & $31,7(1,06)$ & $30,7(1,07)$ \\
Rumanía & $28,2(1,04)$ & $30,9(1,04)$ & $29,8(1,04)$ \\
África & $27,4(1,01)$ & $32,3(1,08)$ & $32,2(1,12)$ \\
Marruecos & $24,9(0,91)$ & $29,8(1,00)$ & $29,6(1,03)$ \\
America Central & $30,8(1,13)$ & $37,7(1,27)$ & $38,0(1,32)$ \\
Rep. Dominicana & $31,7(1,16)$ & $39,8(1,34)$ & $40,2(1,40)$ \\
America del Sur & $31,7(1,16)$ & $37,7(1,27)$ & $36,9(1,29)$ \\
Ecuador & $34,1(1,25)$ & $41,2(1,38)$ & $40,2(1,40)$ \\
Asia & $29,3(1,08)$ & $37,0(1,24)$ & $38,6(1,34)$ \\
China & $32,2(1,18)$ & $39,5(1,33)$ & $39,7(1,38)$ \\
\hline
\end{tabular}

Unidad de superficie: secciones censales

En negrita valores máximos y mínimos para cada año.

Valores relativos en paréntesis; Nacionalidad española $=1,00$

Datos: INE. 
disminuyen. ${ }^{11}$ La calidad geográfica de las áreas residenciales es inferior a la de los ciudadanos españoles cuando los valores son superiores a 1 y viceversa. La fórmula del índice, que representaremos por un $C$, es la siguiente:

(6) $C=\frac{C R E M_{i}+D R M_{i}}{2}$

Siendo $\mathrm{CREM}_{\mathrm{i}}$ la centralización relativa espacial media y $\mathrm{DRM}_{\mathrm{i}}$ la densidad relativa media del grupo $i$.

Los valores para el período 2001-2005, se presentan en la tabla 16.

Tabla 16.

Calidad geográfica en el AMM.

\begin{tabular}{llll}
\hline Nacionalidad & 2001 & 2003 & 2005 \\
\hline Española & 1,000 & 1,000 & 1,000 \\
Extranjera & 0,996 & 1,059 & 1,077 \\
UE-25 & 0,937 & 0,972 & 0,985 \\
UE-15 & $\mathbf{0 , 8 6 5}$ & $\mathbf{0 , 8 9 7}$ & $\mathbf{0 , 9 1 6}$ \\
Europa no-UE & 1,078 & 1,125 & 1,129 \\
Rumanía & 1,089 & 1,130 & 1,129 \\
África & 1,087 & 1,132 & 1,152 \\
Marruecos & 1,073 & 1,119 & 1,136 \\
América Central & 0,991 & 1,059 & 1,084 \\
República Dominicana & 1,000 & 1,083 & 1,108 \\
América del Sur & 0,980 & 1,051 & 1,065 \\
Ecuador & 0,977 & 1,064 & 1,087 \\
Asia & 0,935 & 1,014 & 1,052 \\
China & 0,987 & 1,058 & 1,070
\end{tabular}

En negrita valores máximos y mínimos para cada año.

Valores relativos a la población española. Valores inferiores a 1, mejor calidad geográfica que la población española.

Datos: INE.

${ }^{11}$ Es importante tener en cuenta que para obtener un índice que mediera la calidad global de las áreas residenciales sería necesario tomar, además, otras variables (zonas verdes, calidad de las escuelas, servicios sociales, etc.). Por añadidura, la calidad de los barrios no determina, aunque influye, en la calidad de la vivienda. De hecho, es frecuente que los extranjeros con períodos de residencia largos se trasladen a barrios de peor calidad, a cambio de mejorar la calidad de la vivienda, modificar su tenencia o reducir el número de personas que residen en ella. 
En la tabla 16 podemos advertir que los ciudadanos de la Unión Europea, no sólo presentan los valores más bajos entre los extranjeros, sino que la calidad geográfica de sus barrios es significativamente mejor que la de los españoles. Por otro lado, la calidad geográfica de los barrios de las nacionalidades africanas es la más baja, seguida de las nacionalidades europeas no-UE. Estos resultados sitúan a la población española en un punto intermedio entre las nacionalidades con mayores y menores rentas y apuntan hacia una segregación tanto de tipo "inferior" como "superior".

La conexión de la calidad geográfica y los índices de segregación residencial nos puede ayudar a comprender la relación que existe entre la segregación residencial y la exclusión social. Para ello, podemos comprobar la correlación entre el índice $C$ y el índice $D$-real. Es decir, se trata de comprobar si las nacionalidades más segregadas son también las que residen en barrios con menor calidad geográfica. Con los datos del año 2005, el coeficiente de correlación es 0,72 y estadísticamente significativo con un nivel de confianza del $99 \%$. Por consiguiente, la segregación residencial, con los datos de este estudio, parece estar fuertemente asociada con la calidad geográfica de los barrios.

\section{6) Segregación absoluta}

Una forma interesante de medir la segregación y, posiblemente, la más relevante para medir los cambios en la fisonomía urbana que perciben los ciudadanos, es utilizar los criterios de Johnston et al. (2003) que a su vez recoge ideas del trabajo de Marcuse (1997). Estos criterios clasifican a las áreas residenciales en función del porcentaje de extranjeros residiendo en ellas y del porcentaje que representa una minoría en relación a las demás. El resultado son 6 tipos de áreas residenciales que se describen a continuación:

Ciudadelas: Al menos el $80 \%$ de la población es autóctona.

Comunidades no aisladas: Los autóctonos representan entre el $50 \%$ y el $80 \%$ de la población.

Enclaves pluralistas: Los autóctonos representan una minoría que va desde el $30 \%$ al $50 \%$ de la población.

Enclaves mixtos de minorías: La población autóctona representa menos del $30 \%$ y ninguna minoría representa más del doble que las demás.

Enclaves polarizados: Los autóctonos representan menos del 30\% y una minoría representa el doble que el resto de las minorías juntas.

Guetos: Se cumplen los criterios de los enclaves polarizados, pero además el grupo minoritario más importante representa al menos un $60 \%$ de la población del área residencial y el $30 \%$ del grupo minoritario de toda la ciudad reside en uno de estos guetos.

Utilizando esta clasificación, en la tabla 17 se detallan los datos para el AMM.

Los resultados son sorprendentes puesto que, en todo el período analizado, en ninguna sección censal los extranjeros representan más del $70 \%$ de la población y sólo en una sección censal —o en el 0,03\% de las secciones censales del AMM— las personas 
Tabla 17.

Segregación absoluta en el AMM.

\begin{tabular}{llcc}
\hline \multirow{2}{*}{$\begin{array}{l}\text { Año } \\
\text { (NN secciones) }\end{array}$} & Tipo de enclave & \multicolumn{2}{c}{$\%$ Secciones censales } \\
\hline \multirow{2}{*}{2001 (3778) } & & Criterio de nacionalidad & Criterio de nacimiento \\
& N & 97,22 & 96,06 \\
& PL & 2,75 & 3,92 \\
& MX/PO/GU & 0,00 & 0,03 \\
\multirow{2}{*}{$2003(3814)$} & C & 0,00 & 0,00 \\
& NA & 90,33 & 86,97 \\
& PL & 9,62 & 12,98 \\
& MX/PO/GU & 0,03 & 0,05 \\
& (3896) & 0,00 & 0,00 \\
& NA & 82,57 & 78,23 \\
& PL & 17,40 & 21,71 \\
& MX/PO/GU & 0,03 & 0,05 \\
& C & 0,00 & 0,00 \\
& NA & 81,43 & - \\
& PL & 18,54 & - \\
& MX/PO/GU & 0,03 & - \\
\hline
\end{tabular}

C:Ciudadelas; NA:No aisladas; PL: Pluralistas; MX: Minoritarias-Mixtas; PO: Polarizadas; GU: Guetos.

Unidad de superficie: sección censal.

con nacionalidad española son minoría (a partir del 2003). Estos datos apuntan a la inexistencia de barrios "inmigrantes" y, lógicamente, de guetos étnicos.

Sin embargo, si observamos las diferencias entre el año 2001 y el 2006, la tendencia apunta en la dirección contraria. Progresivamente, las personas con nacionalidad española y las nacidas en España, pierden peso en la población total y, en consecuencia, aumentan el número de comunidades no aisladas y de enclaves pluralistas. Si la tendencia prosigue es previsible que aparezcan en el futuro enclaves mixtos e incluso enclaves polarizados y guetos. Ello dependerá esencialmente del flujo futuro de extranjeros hacia la Comunidad de Madrid y de las pautas que la población extranjera y la población autóctona, especialmente la más joven y móvil, exhiban en el establecimiento del lugar de residencia.

\section{Segregación RESIDENCIAL ECONÓMICA Y CULTURAL}

La distribución territorial de los extranjeros en el AMM no se produce de forma aleatoria. La probabilidad de que una persona con nacionalidad senegalesa, escogido al azar, se instale en el barrio de Salamanca no es equivalente a la probabilidad de que se instale en Alcalá de Henares e igualmente ocurre para una persona con nacionalidad sueca (aunque seguramente con probabilidades muy distintas). De esta distribución no aleato- 
ria surge la segregación residencial, cuyos factores explicativos podemos dividir en dos grandes bloques: segregación residencial económica y cultural.

\section{Segregación residencial económica}

Tradicionalmente, los estudiosos prestaron mayor atención a la segregación producida por las desigualdades económicas. Hoy, la universalización de los movimientos migratorios ha restado preeminencia al estudio de este tipo de segregación, si bien esto no impide que, la segregación urbana de los inmigrantes pueda deberse principalmente a diferencias de poder adquisitivo y no a supuestas diferencias culturales.

La segregación residencial económica se origina por medio de dos mecanismos, cuyo supuesto esencial compartido es la existencia de desigualdad económica.

El primero de los mecanismos se basa en la existencia de preferencias compartidas, por la mayoría de la población, sobre las características deseables que debe poseer una zona residencial como pueden ser servicios sociales de calidad, escuelas con buenos resultados académicos, amplias zonas verdes o la cercanía al centro urbano. Si los supuestos se cumplen - existe desigualdad económica y existen preferencias residenciales compartidas - se producirá un mapa residencial en el que los grupos con mayores rentas ocuparán las zonas residenciales privilegiadas y los de menores rentas los barrios menos deseados. Por otra parte, debido a que la segregación residencial es relativa a la distribución territorial de la población mayoritaria, este mecanismo producirá dos tipos de segregación residencial en función del diferencial de renta con los españoles: segregación "inferior", de nacionalidades con ingresos inferiores a los españoles en lugares de calidad inferior como Vallecas o Coslada y segregación "superior", de nacionalidades con ingresos superiores a los españoles en lugares de mayor calidad como el barrio de Salamanca o el municipio de Las Rozas.

El segundo mecanismo está relacionado con la teoría de la discriminación estadística, introducida por Phelps (1972) y Arrow (1972). En su versión más simple, esta teoría afirma que las personas que aspiran a un empleo, cuyas habilidades y capacidades relevantes no son directamente observables, serán discriminadas en base a otras características que sí son observables como el grupo étnico, el origen social o el lugar de residencia. Extendiendo el argumento al caso que nos ocupa, el poder adquisitivo del vecindario, al ser un hecho fácilmente observable, sirve para predecir comportamientos más o menos deseables del vecindario. Un corolario de este argumento es que los individuos con mayores rentas estarán dispuestos a pagar un sobreprecio por residir en vecindarios de elevado poder adquisitivo, aumentando el precio de la vivienda y evitando la llegada de personas con menores rentas. 


\section{Segregación residencial cultural}

El principal mecanismo que sustenta este tipo de segregación es la búsqueda de una mayor "comodidad" cultural por parte de los individuos, lo que se traduce en preferencias por vecindarios donde una proporción significativa es de la misma comunidad cultural, grupo étnico o nacionalidad. El modelo más elaborado de este tipo de segregación, el cual ha sido demostrado empíricamente por Clark (1991), se lo debemos a Schelling (1971). Schelling investiga, por medio de modelos con dos grupos étnicos, las consecuencias que, para el "ajedrez" residencial, tiene la distribución de las preferencias individuales sobre la composición étnica de los vecindarios. Su primer hallazgo muestra que, si la distribución de preferencias individuales sobre la composición étnica de los vecindarios cumple determinados criterios no excesivamente estrictos, existirá un punto o momento (tipping point) a partir del cual la segregación de un barrio será imparable. El segundo hallazgo revela que para que la segregación cultural ocurra no es necesario que la mayoría de individuos posea preferencias extremistas. De hecho, sobrepasado cierto umbral, la radicalidad de las preferencias afectará a la velocidad del proceso segregador pero no al resultado final. Por lo tanto, es importante tener en cuenta que preferencias relativamente moderadas sobre la composición étnica del barrio, como pueden ser vecindarios donde el propio grupo represente un cuarto de la población, pueden generar mapas residenciales altamente segregados.

\section{Tiempo de residencia y renta}

El tiempo de residencia diversifica las redes sociales de los extranjeros y tiende a mejorar y estabilizar su situación socioeconómica. Por otra parte, a medida que los extranjeros se integran en la sociedad receptora el conocimiento sobre el mercado inmobiliario local mejora considerablemente, permitiendo a los individuos elegir zonas residenciales con una mejor relación calidad/precio. Todo ello debería contribuir a que, a medio y largo plazo, la renta se convierta en el factor explicativo principal de la segregación residencial de los extranjeros y que los rasgos culturales pierdan relevancia.

Si esta hipótesis es cierta, a medio y largo plazo, las nacionalidades con mayores rentas harán valer su poder económico en el mercado residencial y acabarán concentrándose en los barrios de mayor calidad. Por el contrario, las nacionalidades con menores rentas, en las cuales la desigualdad económica intragrupal acostumbra a aumentar durante el proceso integrador, tenderán a diversificar sus lugares de residencia, disminuyendo su segregación residencial.

Para comprobar la validez de esta hipótesis, en el modelo se interaccionan las variables sustitutas de la renta y el tiempo de residencia. Si el coeficiente es positivo, podemos afirmar que el tiempo de residencia aumentará (disminuirá) la segregación residencial de las nacionalidades menos pudientes en menor (mayor) medida que para las nacionalidades con mayor poder adquisitivo. 


\section{Metodología}

Para comprobar las distintas hipótesis, he llevado a cabo tres modelos de regresión lineal múltiple, utilizando el método de los mínimos cuadrados ordinarios (MCO). El primero de ellos pretende comprobar, de forma parsimoniosa, el efecto de la renta y el tiempo de residencia. El segundo modelo tiene como objetivo separar la segregación residencial cultural de la económica, así como comprobar el efecto de la interacción entre el tiempo de residencia y la renta. Esta interacción nos ofrece información sobre la variación temporal de la segregación residencial en función de la renta de las distintas nacionalidades. El último modelo es idéntico al anterior pero utilizando, como variable sustituta de la renta, los datos sobre ocupación de la Encuesta Población Activa (EPA), más fiables pero con datos para menos nacionalidades.

\section{Los datos}

Dos advertencias previas sobre las deficiencias de los datos, y que nos obligan a tomar los resultados con precaución, son necesarias. En primer lugar, al utilizar principalmente datos agregados no es posible distinguir adecuadamente entre los distintos mecanismos causales que se esconden detrás de las correlaciones. En segundo lugar, algunas de las variables utilizadas en los modelos son sustitutas (proxies), existiendo la posibilidad de que no estén midiendo lo que se pretende que midan. Este riesgo es especialmente importante en los dos primeros modelos que, sin embargo, nos permiten trabajar con un mayor número de casos.

Para los dos primeros modelos, se han utilizado los datos de las 46 nacionalidades, que en el año 2005, superaban los 1.000 habitantes en el AMM. En el Modelo 3 se han incluido 27 nacionalidades, eliminando todas aquellas con menos de 20 casos en los datos sobre ocupación de la EPA (primer trimestre, 2005). ${ }^{12}$ La variable dependiente es el índice de uniformidad en el año 2005, obtenido en la primera parte del estudio. Las variables independientes son las siguientes:

- LN_POB_2005: ${ }^{13}$ Logaritmo neperiano de la población del grupo en el año 2005. Es una variable de control que permite controlar el efecto que el tamaño de la población ejerce sobre el índice de uniformidad, ajustándolo a los valores reales de segregación. Controlar por esta variable permite obtener el índice D-real. Los valores elevados de $\mathrm{R}^{2}$ se deben principalmente a esta variable.

- POB_98/05: Población del grupo en el año 1998, dividida entre la población del año 2005. Es una variable sustituta (proxy), que pretende medir el tiempo de residen-

\footnotetext{
${ }^{12} \mathrm{He}$ llevado a cabo el mismo análisis de regresión con aquellas nacionalidades con más de 5, 10 y 25 casos en los datos EPA y los resultados son prácticamente idénticos. Finalmente, he optado por el criterio de los 20 casos porque es el criterio más restrictivo, manteniendo, al menos, dos casos por región geográfica.

${ }^{13}$ Más información sobre esta variable en el apéndice.
} 
cia en la sociedad de recepción. La EPA también incluye una variable sobre el tiempo de residencia. Sin embargo, el escaso número de casos para la provincia de Madrid obliga a utilizar totales nacionales, aumentando el riesgo de falseamiento en los datos. De todas formas, ambas variables están significativamente correlacionadas $(0,77)$ y los resultados no varían significativamente al emplear una u otra variable.

- CSE (EPA): Variable derivada de la "Asignación de la condición socioeconómica" de la EPA (2005). Se han reducido las categorías a cuatro: trabajadores no cualificados y otros empleados (1), profesionales y técnicos (2), directivos (3) y empresarios (4). Se han excluido a los profesionales de las FF.AA. por su difícil encaje. Los datos muestran totales nacionales.

- LN_RENTA: Logaritmo neperiano de la renta per capita del país de origen (Banco Mundial, 2005). Es una variable sustituta que pretende medir la renta en Madrid de los distintos grupos. Al tomar los logaritmos, las diferencias entre las distintas nacionalidad se reducen y se ajustan más a la distribución de la renta para el caso madrileño. Cuanto más representativos sean los extranjeros de la población de su país de origen, mejor sustituirá esta variable a la situación económica en España. A pesar del riesgo de falseamiento asociado a esta variable, la elevada correlación con $\operatorname{CSE}(E P A)(0,63)$ nos aporta seguridad de que no se desvía en exceso de la variable que pretende sustituir.

- UE-15/EU_POST/AMÉRICA/ ÁFRICA/ASIA: Variables dicotómicas que identifican la zona geográfica de donde provienen los grupos de extranjeros. Pretenden medir la influencia de los rasgos culturales en el índice de uniformidad. Las nacionalidades de la UE-15, son la referencia para medir el efecto del resto de nacionalidades.

\section{Resultados}

El primer resultado relevante es que el efecto de la variable de control $L N \_P O B \_2005$ es semejante en los tres modelos, lo que corrobora el potencial comparativo del índice $D$-real señalado más arriba. Otro resultado a tener en cuenta es la similitud entre los resultados del segundo y tercer modelo, variando de forma relevante sólo los coeficientes del tiempo de residencia. Asimismo, la significatividad estadística de los coeficientes es similar en ambos modelos, y ello a pesar de que el modelo con datos de la EPA utiliza datos sólo para 27 nacionalidades. Esta semejanza confiere mayor robustez a los resultados globales.

En cuanto a los resultados específicos de las variables, el primer modelo apunta a un efecto depresor de la renta sobre la segregación residencial, resultado corroborado tanto por el segundo modelo como por el tercero. Estos resultados nos permiten afirmar que la segregación residencial está negativamente asociada con la renta de las nacionalidades extranjeras. El efecto del tiempo de residencia es más ambiguo. En los modelos 2 y 3 , los coeficientes son significativos al $15 \%$ pero con distintos signos. Esto nos impide establecer de forma clara el efecto del tiempo de residencia. Los resultados son más concluyentes en el caso de la interacción entre renta y tiempo de residencia. Si bien esta 
Tabla 18.

Análisis multivariante MCO. Determinantes de la segregación residencial de los extranjeros en el AMM, año 2005.

\begin{tabular}{|c|c|c|c|}
\hline Variables & Modelo 1 & Modelo 2 & Modelo 3 \\
\hline CONSTANTE & $\begin{array}{l}2,059^{\star * *} \\
19,22\end{array}$ & $\begin{array}{l}1,861^{* * *} \\
11,60\end{array}$ & $\begin{array}{l}0,822^{* * *} \\
3,81\end{array}$ \\
\hline $\begin{array}{l}\text { Variable de control } \\
\text { LN_POB_2005 }\end{array}$ & $\begin{array}{l}-0,110^{* * *} \\
-12,77\end{array}$ & $\begin{array}{l}-0,096^{* * *} \\
-13,67\end{array}$ & $\begin{array}{l}-0,094^{* * *} \\
-10,41\end{array}$ \\
\hline $\begin{array}{l}\text { Tiempo de residencia } \\
\text { POB_98/05 }\end{array}$ & $\begin{array}{l}0,025 \\
0,39\end{array}$ & $\begin{array}{l}-0,626 \\
-1,61\end{array}$ & $\begin{array}{l}0,842 \\
1,61\end{array}$ \\
\hline $\begin{array}{l}\text { Situación económica } \\
\text { LN_RENTA }\end{array}$ & $\begin{array}{l}-0,052^{* * *} \\
-3,86\end{array}$ & $\begin{array}{l}-0,045^{* *} \\
-2,22\end{array}$ & \\
\hline CSE (EPA) & & & $\begin{array}{l}-0,188^{\star * *} \\
-3,00\end{array}$ \\
\hline $\begin{array}{l}\text { Interacción } \\
\text { LN_RENTA * POB_98/05 }\end{array}$ & & $\begin{array}{l}0,064 \\
1,43\end{array}$ & \\
\hline CSE (EPA) * POB_98/05 & & & $\begin{array}{l}0,285^{*} \\
1,86\end{array}$ \\
\hline $\begin{array}{l}\text { Rasgos culturales (UE-15 r } \\
\text { EU_POST }\end{array}$ & & $\begin{array}{l}0,026 \\
0,66\end{array}$ & $\begin{array}{l}-0,018 \\
-0,29\end{array}$ \\
\hline AMÉRICA & & $\begin{array}{l}-0,030 \\
-0,93\end{array}$ & $\begin{array}{l}-0,040 \\
-0,81\end{array}$ \\
\hline ÁFRICA & & $\begin{array}{l}0,085^{* *} \\
2,33\end{array}$ & $\begin{array}{l}0,106^{*} \\
1,86\end{array}$ \\
\hline ASIA & & $\begin{array}{l}0,121^{\text {***}} \\
3,46\end{array}$ & $\begin{array}{l}0,191^{* * *} \\
3,11\end{array}$ \\
\hline $\mathrm{R}^{2}$ corregida & 0,854 & 0,918 & 0,889 \\
\hline Observaciones & 46 & 46 & 27 \\
\hline
\end{tabular}

En negrita los valores del estadístico $t$

${ }^{* * *}$ significativo al nivel $1 \%$

${ }^{* *}$ significativo al nivel $5 \%$

*significativo al nivel $10 \%$ 
variable es sólo significativa en el tercer modelo, el signo del coeficiente se mantiene constante entre modelos. El signo positivo indica que el efecto depresor de la renta disminuirá con el tiempo de residencia o, alternativamente, que el efecto positivo del tiempo de residencia sobre la segregación residencial del Modelo 3 será menor para los grupos con menores rentas. Este resultado confirma, parcialmente, la hipótesis sobre la interacción que habíamos establecido previamente. Por último, las variables dicotómicas regionales nos permiten afirmar que la segregación residencial está asociada tanto con aspectos culturales como económicos, siendo los grupos más alejados culturalmente de España (Asia y África) los que mayores coeficientes presentan. Sorprendentemente, el coeficiente de las nacionalidades americanas es inferior al de las nacionalidades de la UE-15, si bien éste no es estadísticamente significativo.

\section{CONCLUSIÓN}

En este trabajo, el lugar de residencia ha sido considerado como un espacio de socialización, en el cual los individuos establecen y mantienen redes sociales que les habilitan o limitan en sus posibilidades de progresión socioeconómica. Estas redes se superponen en muchas ocasiones a las que los individuos poseen en otros ámbitos, como la escuela y el trabajo, lo que, muy a menudo, encierra a los individuos en un círculo de relaciones relativamente homogéneas. Esta homogeneidad facilita la estabilidad en la situación socioeconómica de la población urbana puesto que las redes animan las acciones de los individuos a través del "efecto imitación" e influyen decisivamente en su participación en el mercado laboral, y eventualmente en el formativo-educativo y en el residencial. El "barrio" es, por tanto, un espacio relevante en la aparición de la exclusión social, entendiendo por exclusión social la ausencia de oportunidades o la incapacidad de determinados individuos de materializar su potencial como miembros de una sociedad.

Una manera de identificar la probabilidad de que las redes sociales entabladas por un grupo sean homogéneas es medir su segregación residencial, objetivo prioritario de este trabajo. Los resultados, respecto a este objetivo, han sido satisfactorios, si bien en algunos aspectos será necesario afinar el análisis mediante datos de tipo individual, como los utilizados por Arjona y Checa (2006) para el caso almeriense.

Los resultados más destacados del trabajo son los siguientes:

- La renta está negativamente asociada con la segregación residencial de los extranjeros, asociación que se debilita con el tiempo de residencia.

- El efecto del tiempo de residencia es ambiguo y estadísticamente poco significativo. Controlando por la renta y el tiempo de residencia, las nacionalidades más segregadas son las africanas y las asiáticas, posiblemente debido a su "alejamiento cultural" de la población autóctona.

- No existen guetos en el AMM, si por tal entendemos secciones censales donde la población autóctona represente menos del 30\%. Sin embargo, las ciudadelas, o secciones censales donde al menos el $80 \%$ de la población es autóctona, están en franco 
retroceso desde el $97 \%$ del total de secciones censales en 2001 hasta el $81 \%$ en 2006 .

- La calidad geográfica de los barrios está fuertemente asociada con la segregación residencial. Es decir, las nacionalidades que residen en barrios con menor calidad geográfica son también las más segregadas residencialmente.

- Las nacionalidades de la UE-15 son las mejor distribuidas a lo largo del AMM, así como las más expuestas a la población española. Además, residen en barrios con mejor localización que el resto de nacionalidades, incluyendo a la española, lo que apunta a la existencia de una moderada segregación de nacionalidades con elevado poder adquisitivo.

- En el lado opuesto, las nacionalidades peor distribuidas, tanto por municipios como por secciones censales, son las africanas las cuales se encuentran, en términos relativos, poco expuestas a la población española. Asimismo, la calidad geográfica de las zonas urbanas donde residen es la más baja puesto que se encuentran lejos del centro y, en ellas, la densidad de población es elevada. Su segregación, siguiendo el análisis econométrico, viene explicada tanto por diferencias culturales como por desigualdades económicas.

- Las nacionalidades americanas presentan valores intermedios en todos los índices si bien se aprecia un incremento de la segregación a lo largo del tiempo, un empeoramiento de la calidad geográfica de los barrios y una disparidad elevada entre las distintas nacionalidades (Argentina en un extremo y Ecuador en el otro). El análisis econométrico sugiere que los factores culturales reducen su segregación y los económicos la aumentan.

- En cuanto a las nacionalidades asiáticas, su distribución en el AMM es muy desigual, su exposición a la población española es la más baja y, sin embargo, residen en barrios de calidad geográfica media. El análisis econométrico apunta a que su segregación viene explicada principalmente por diferencias culturales.

- Las nacionalidades europeas no UE-15 presentan valores de segregación moderados, segregación que viene explicada por la alta concentración de población rumana en los municipios del este de la Comunidad de Madrid. Las diferencias económicas con la población española explican sus niveles de segregación residencial.

- Desde el punto de vista metodológico, se ha demostrado la ineficacia comparativa del índice de uniformidad, comúnmente utilizado en la literatura y se ha propuesto un nuevo índice ( $D$-real), basado en el anterior, con capacidad comparativa entre grupos.

\section{REFERENCIAS BibLIOGRÁFICAS}

Arrow, K. 1972. Racial Discrimination in Economic Life. Lexington MA: Lexington Books.

Checa Olmos, J. C. y A. Arjona Garrido. 2006. "Inmigración y segregación residencial. Aproximación teórica y empírica para el caso almeriense". Migraciones. 20: 143-171.

Clark, W.A.V. 1991. "Residential Preferences and Neighborhood Racial Segregation: A Test of the Schelling Segregation Model". Demography. 28: 1-19. 
Goldberg, D. T. 1998. "The New Segregation". Race and Society. 1: 15-32.

Goldhaber, R. y I. Schnell. 2007. "A Model of Multidimensional Segregation in the Arab Ghetto in Tel Aviv-Jaffa". Tijdsschrift voor Economische en Sociale Geografie. 98: 603-620.

Johnston, R., M. Poulsen y J. Forrest. 2003. "And did the walls come tumbling down? Ethnic residential segregation in four U.S. metropolitan areas 1980-2000". Urban Geography. 24: 560-581.

Marcuse, P. 1997. "The Enclave, the Citadel, and the Ghetto". Urban Affairs Review. 33: 228-264.

Martínez Veiga, U. 1999. Pobreza, segregación y exclusión social. La vivienda de los inmigrantes extranjeros en España. Barcelona: Icaria.

Massey, D. 1996. "The Age of Extremes: Concentrated Affluence and Poverty in the Twenty-First Century". Demography. 33: 395-412.

Massey, D. y N. Denton. 1988. "The Dimensions of Residential Segregation". Social Forces. 67: 281 315.

Maseey, D. y N. Denton. 1993. American Apartheid. Cambridge, Mass.: Harvard University Press.

Musterd, S. y W. Ostendorf. 2007. "Spatial Segregation and Integration in the Netherlands". En Residential Segregation and the Integration of Immigrants: Britain, the Netherlands and Sweden, Schönwälder, K. coord., WZB, WP 2007-602.

Phelps, E. 1972. "The Statiscal Theory of Racism and Sexism". The American Economic Review. 62: 659-661.

Schelling, T. 1971. "Dynamic models of segregation”. Journal of Mathematical Sociology .1: 143-186.

Wilson, W. 1987. The Truly Disadvantaged: The Inner City, Underclass, and Public Policy. Chicago: University of Chicago Press.

Wilson, W. 1996. When Work Disappears: The World of the New Urban Poor. Nueva York: Knopf.

\section{Fuentes de datos y otras referencias}

Datos del Padrón Continúo. www.ine.es

Encuensta de Población Activa (EPA) (1er trimestre, 2005). www.ine.es

Notas de selectividad por centros educativos. www.magisnet.com (última consulta: 01/10/2007)

World Development Indicators (2005). Banco Mundial. http://devdata.worldbank.org/wdi2005/Cover.htm

Comisión Europea (2003), Comunicación sobre Inmigración, integración y empleo, COM (2003) 336. 


\section{APÉNDICE}

\section{Método de obtención de D-real}

El problema del índice de disimilitud o uniformidad consiste en su dependencia del tamaño de la población. Por esta razón, es necesario corregir por el tamaño de la población de los grupos por medio del análisis multivariante y utilizando posteriormente los residuos para conseguir una medición más ajustada de la segregación residencial en la dimensión de la uniformidad. Un problema añadido es que la relación de la población con el índice $D$ no es lineal sino curvilínea, como se puede observar en el primer gráfico. El logaritmo neperiano de la población, al representar el índice $D$ la población en porcentaje, soluciona el problema, convirtiendo la relación de la población y el índice en lineal, como se puede apreciar en el Gráfico 2. Por lo tanto, el índice D-real no es más que los residuos de una regresión donde la variable dependiente es el índice de uniformidad (ID_2005) y la variable independiente es el logaritmo neperiano de la población (LN_2005).

Gráfico 1.

Índice de uniformidad y población.

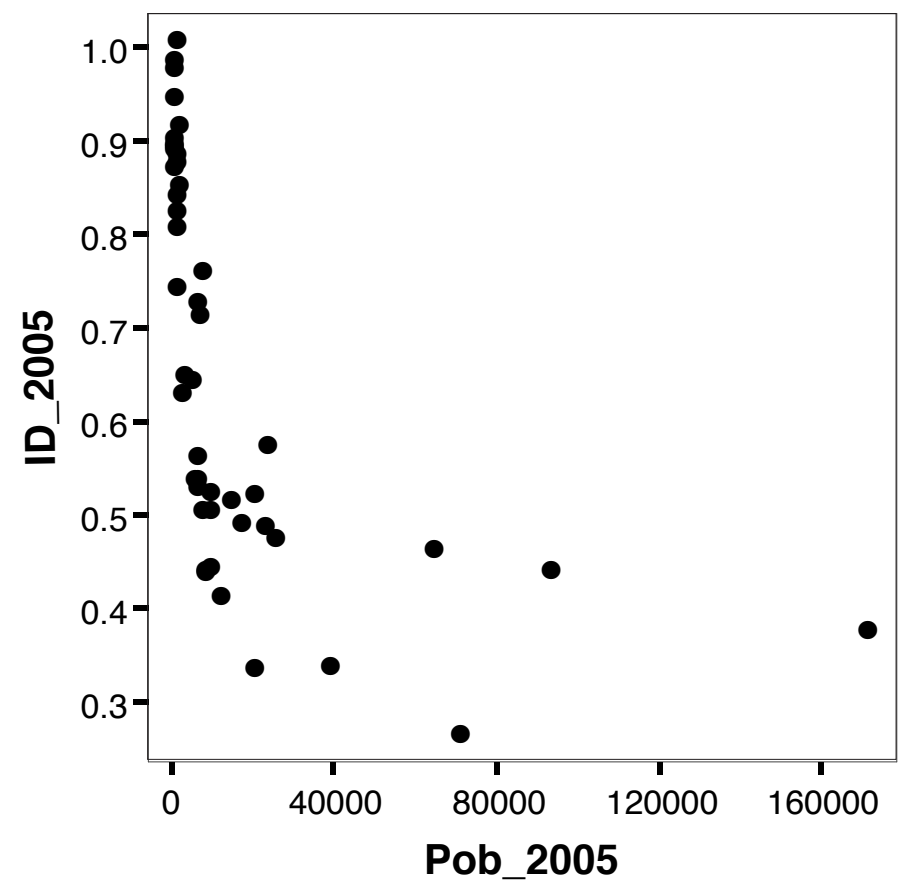




\section{Gráfico 2.}

Índice de uniformidad y logaritmo de la población

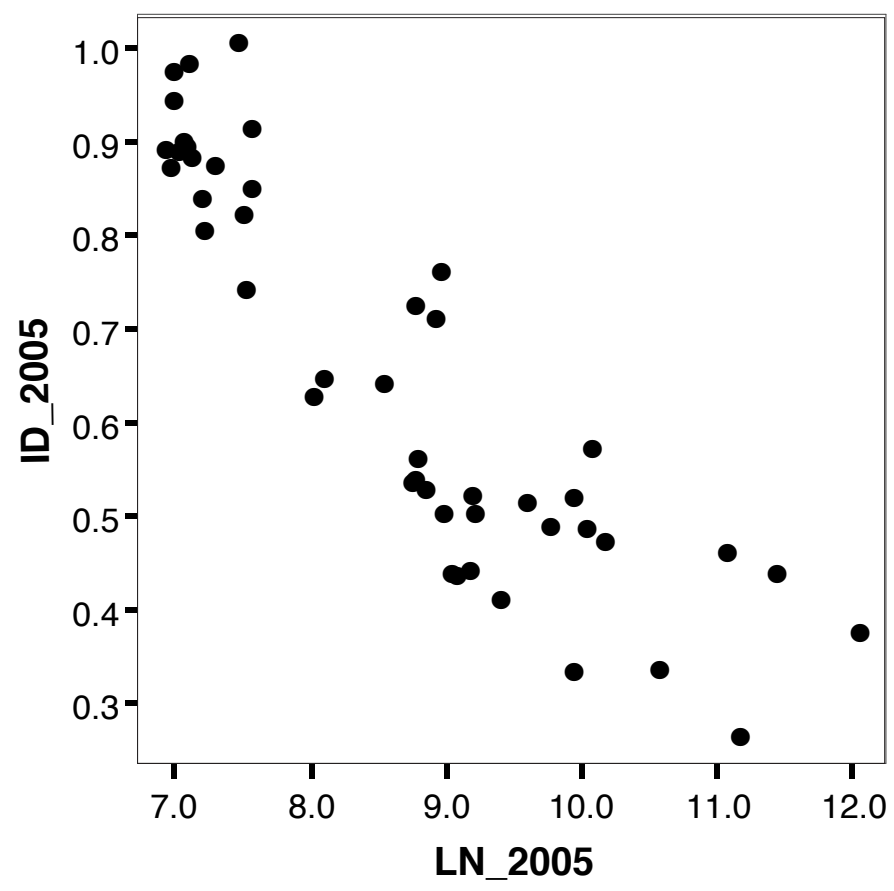

ALFONSO ECHAZARRA es estudiante de doctorado en el Centre for Census and Survey Research (CCSR) de la Universidad de Manchester. Hasta el momento sus investigaciones han girado en torno al fenómeno migratorio, ya sea en su vertiente económica (remesas de inmigrantes), demográfica (segregación residencial) o social (delincuencia).

RECIBIDO: $12 / 11 / 07$

ACEPTADO: 26/11/08

Publicado on-line: 18 de diciembre de 2009 